\title{
Recent Trends of Computational Methods in Vibration Problems
}

\author{
Snehashish Chakraverty, ${ }^{1}$ Atma Sahu, ${ }^{2}$ Choong Kok Keong, ${ }^{3}$ and Saleh M. Hassan ${ }^{4}$ \\ ${ }^{1}$ Department of Applied Mathematics, National Institute of Technology, Rourkela 769 008, India \\ ${ }^{2}$ Department of Mathematics, Coppin State University, Baltimore, MD 21216, USA \\ ${ }^{3}$ School of Civil Engineering, Universiti Sains Malaysia, Engineering Campus Seri Ampangan, 14300 Nibong Tebal, Malaysia \\ ${ }^{4}$ Department of Mathematics, College of Sciences, King Saud University, P.O. Box 2455, Riyadh 11451, Saudi Arabia
}

Correspondence should be addressed to Snehashish Chakraverty, sne_chak@yahoo.com

Received 23 October 2011; Accepted 23 October 2011

Copyright () 2012 Snehashish Chakraverty et al. This is an open access article distributed under the Creative Commons Attribution License, which permits unrestricted use, distribution, and reproduction in any medium, provided the original work is properly cited.

Prior to developing expensive prototype systems in engineering and sciences, it is well known that computational methods are often called into inquiry. Therefore, in order to allow prototyping of engineering systems to be in greater confidence, the degree of correlation between computational modeling predictions and experimental results has to be adequate. Accordingly, new and efficient computational methods help in determining the validation of the experimental results giving rise to a reliable and timely design systems.

The aim of this special issue is to bring together the leading researchers of dynamics and vibration area including applied mathematicians, and allow them to share their original research work. Mathematical theory, numerical simulation, physical experiments with advanced computational investigations, engineering design, and their various engineering applications are included in the main program of the issue.

Accordingly, various papers on vibration analysis have been included in this special issue after completing a careful rigorous peer-review process. In particular, a new actuator for reducing rotor vibrations in electrical machines is investigated in one of the papers. In addition to the traditional prediction error method, a new knowledge-based artificial Fish-Swarm optimization algorithm (AFA) with crossover, CAFAC, is proposed to identify the parameters in the new model. The prediction error method (PEM) is also employed to identify the induction motor to produce a black box model with correspondence to input-output measurements.
Another paper is of use of particle Swarm optimization to find the best teeth modifications for multimesh helical gears, which are crucial for the static transmission error (STE). Robustness of the solutions toward manufacturing errors and applied torque is analyzed by the particle Swarm algorithm to access to the deterioration capacity of the tested solution.

In another paper, the homotopy analysis method (HAM) is employed to propose a highly accurate technique for solving strongly nonlinear aeroelastic systems of airfoils in subsonic flow. The frequencies and amplitudes of limit cycle oscillations (LCOs) arising in the considered systems are expanded as a series of an embedding parameter. Numerical examples show that the HAM solutions are obtained very precisely.

Then stochastic BEM has been used to handle geometrical uncertainties within the classic BEM formulation. As a result, the solution shows deterministic behaviour at low frequencies; decreasing the wavelength, the effect of the uncertainties smooths the response.

In order to assess the dynamic behavior of the highspeed vibratory drilling system, this study develops a rotorbased finite element model, integrated with the modelling of component interfaces. The current results indicate that the simulations are consistent with the experimental measurements.

The automotive gearbox rattle noise resulting from vibro-impacts that can occur between the idle gears under excessive velocity fluctuations of the shaft-driving gears imposed by engine torque fluctuation is also studied. This 
study presents various effect components on the dynamic response of the idle gear.

As of today, vibration analysis of complex systems is commonly encountered in science and engineering practice. Analysis and design of such structures call for efficient computational tools. As such, the present issue has addressed recent trends of the computational methods that may be used in the said vibration problems. Finally, it is hoped that the present special issue would certainly ignite researchers new problem domain and enhance efficiency and accuracy of the solution methods in use to-day.

\section{Snehashish Chakraverty \\ Atma Sahu \\ Choong Kok Keong \\ Saleh M. Hassan}



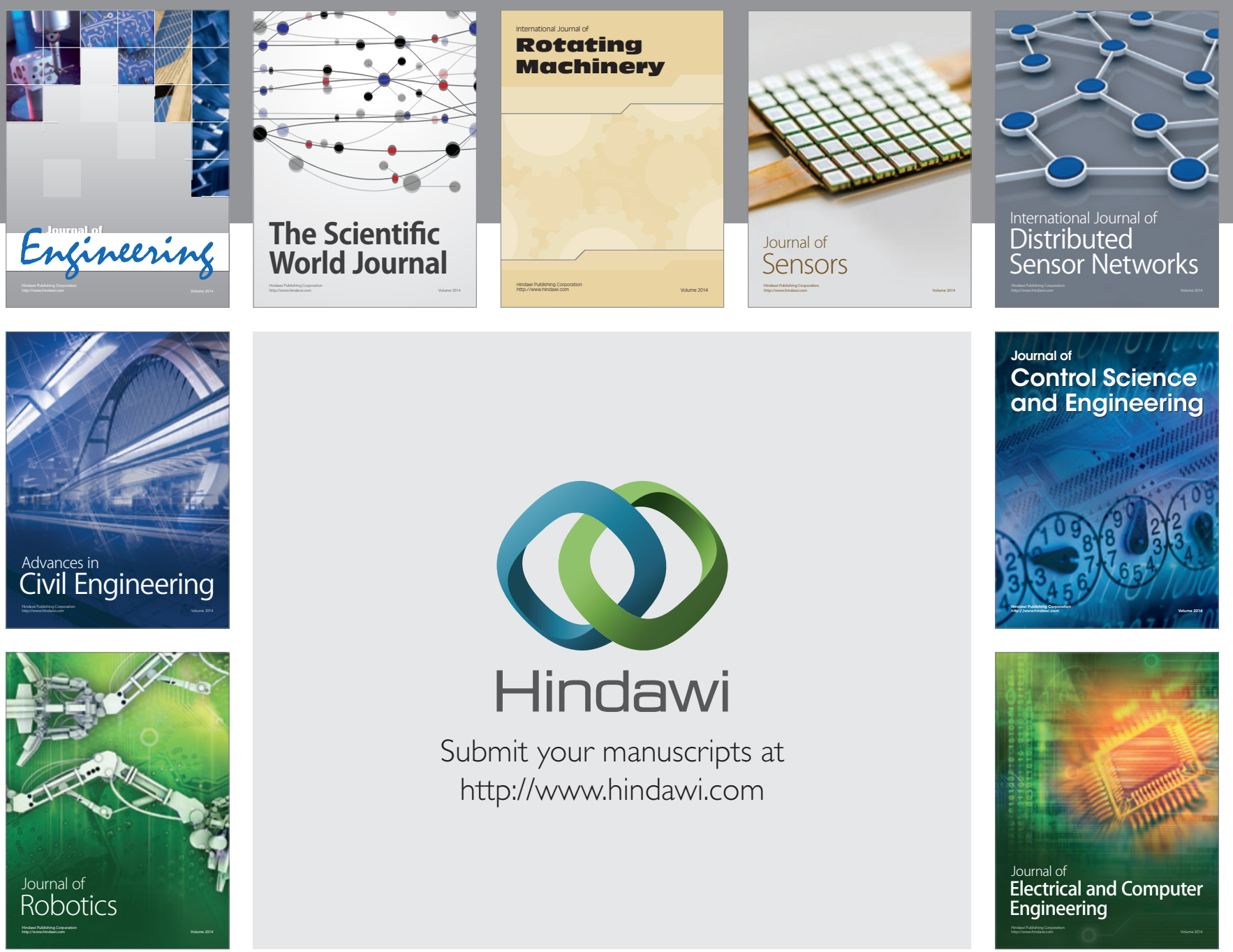

Submit your manuscripts at

http://www.hindawi.com
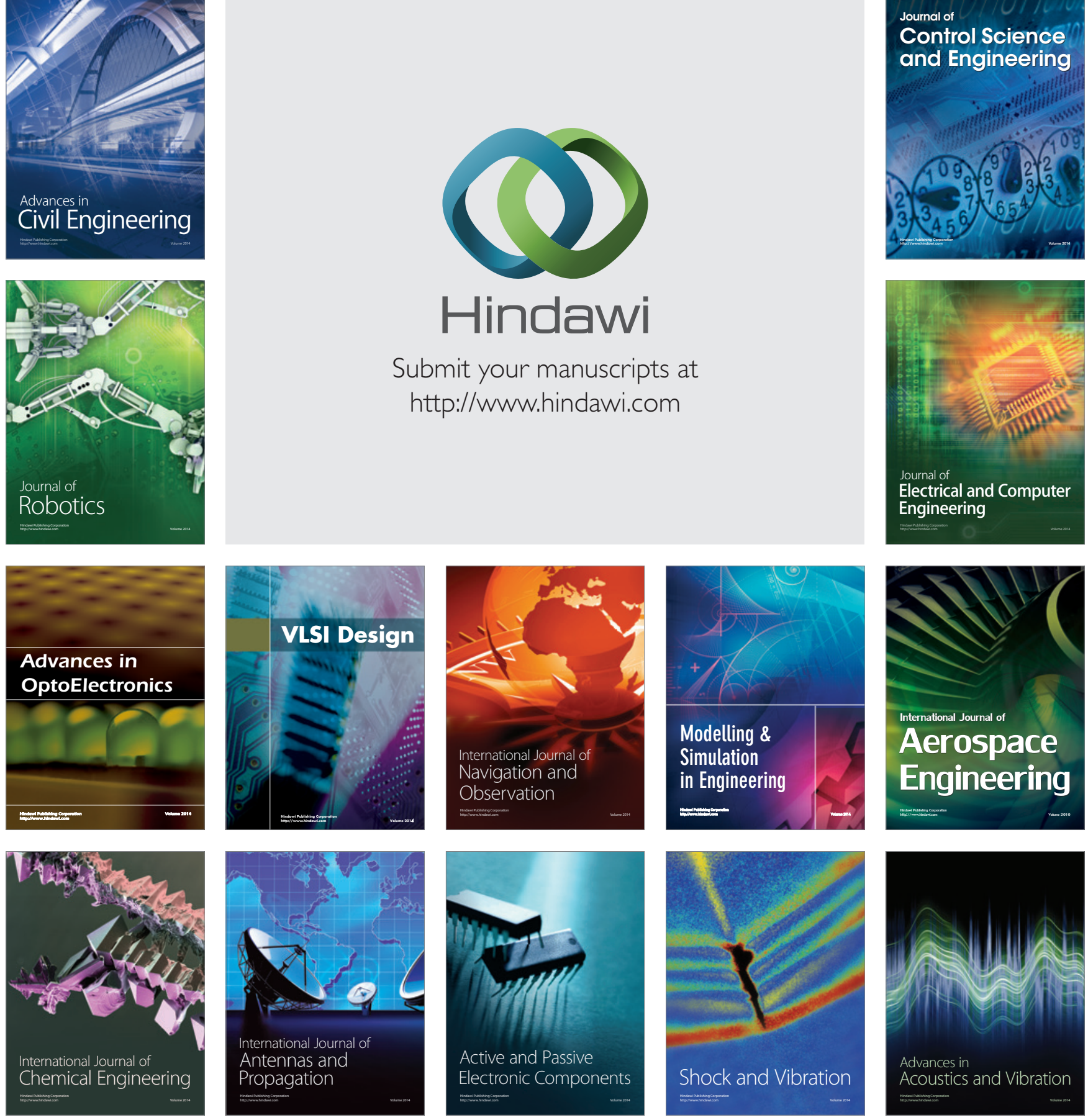